\title{
A description of a tailored knowledge translation intervention delivered by knowledge brokers within public health departments in Canada
}

Maureen Dobbins ${ }^{1 *}$ (D) Lori Greco ${ }^{2}$, Jennifer Yost ${ }^{3}$, Robyn Traynor ${ }^{4}$, Kara Decorby-Watson ${ }^{5}$ and Reza Yousefi-Nooraie ${ }^{6}$

\begin{abstract}
Background: While there is an expectation to demonstrate evidence-informed public health there is an ongoing need for capacity development. The purpose of this paper is to provide a description of a tailored knowledge translation intervention implemented by knowledge brokers (KBs), and reflections on the factors that facilitated or hindered its implementation.

Methods: The 22-month knowledge translation intervention, implemented by two KBs, sought to facilitate evidence-informed public health decision-making. Data on outcomes were collected using a knowledge, skills and behavioural assessment survey. In addition, the KBs maintained reflective journals noting which activities appeared successful or not, as well as factors related to the individual or the organisation that facilitated or hindered evidence-informed decision-making.

Results: Tailoring of the knowledge translation intervention to address the needs, preferences and structure of each organisation resulted in three unique interventions being implemented. A consistent finding across organisations was that each site needed to determine where evidence-informed decision-making 'fit' within pre-existing organisational processes. Components of the intervention consistent across the three organisations included one-to-one mentoring of teams through rapid evidence reviews, large group workshops and regular meetings with senior management. Components that varied included the frequency of the KB being physically onsite, the amount of time staff spent with the KB and proportion of time spent one-to-one with a KB versus in workshops. Key facilitating factors for implementation included strong leadership, influential power of champions, supportive infrastructure, committed resources and staff enthusiasm.

Conclusions: The results of this study illustrate the importance of working collaboratively with organisations to tailor knowledge translation interventions to best meet unique needs, preferences, organisational structures and contexts. Organisational factors such as leadership, champions and supportive infrastructure play a key role in determining the impact of the knowledge translation interventions. Future studies should explore how these factors can be fostered and/or developed within organisations. While KBs implemented the knowledge translation intervention in this study, more research is needed to understand the impact of all change agent roles including KBs, as well as how these roles can be maintained in the long-term if proven effective.
\end{abstract}

Keywords: Evidence-informed decision-making, public health, knowledge translation, capacity-building, knowledge broker

\footnotetext{
* Correspondence: dobbinsm@mcmaster.ca

${ }^{1}$ Faculty of Health Sciences, School of Nursing, McMaster University, 175

Longwood Road South, Suite 210A, Hamilton, Ontario, Canada

Full list of author information is available at the end of the article
}

(c) The Author(s). 2019 Open Access This article is distributed under the terms of the Creative Commons Attribution 4.0 International License (http://creativecommons.org/licenses/by/4.0/), which permits unrestricted use, distribution, and reproduction in any medium, provided you give appropriate credit to the original author(s) and the source, provide a link to the Creative Commons license, and indicate if changes were made. The Creative Commons Public Domain Dedication waiver (http://creativecommons.org/publicdomain/zero/1.0/) applies to the data made available in this article, unless otherwise stated. 


\section{Background}

Public health professionals in Canada are expected to deliver effective and efficient programmes and services that protect and promote the health of the population [1]. Evidence-informed decision-making (EIDM) is one component of public health decision-making that can support the implementation of effective and efficient programmes and services. EIDM is defined as the incorporation of the best available research evidence along with knowledge related to the setting, political and societal preferences, resources and public health expertise into decisions $[2,3]$. The National Collaborating Centre for Methods and Tools (NCCMT) advocates that EIDM is comprised of seven steps, namely 'define' (a searchable question), 'search' (for evidence), 'appraise' (the quality of evidence), 'synthesise' (the evidence), 'adapt' (to the local context), 'implement' (a change in practice) and 'evaluate' (impact) [4]. Research has shown EIDM is associated with more effective policies and programmes being implemented, as well as optimal use of scarce public health resources $[2,5]$.

While the public health sector in Canada has been working toward EIDM for many years [6], knowledge translation (KT) interventions are still needed to support public health organisations in developing the culture, context and infrastructure to support EIDM, as well as to assist public health professionals in obtaining the knowledge, skills and capacity necessary to practice in an evidence-informed way [7, 8]. Research has shown that early decision-maker involvement in the research process, messages tailored to decision-maker needs and attention to context help facilitate EIDM [9]. Research also suggests that activities that address both context and individual capacity hold promise for achieving EIDM [10, 11]. While many KT interventions have been evaluated, questions still remain as to how best to support EIDM in public health.

Knowledge brokers (KB) are one type of change agent that may be used to implement $\mathrm{KT}$ interventions. KBs work to promote, facilitate and support EIDM through one-to-one interaction with individuals, teams and organisations $[12,13]$. One way in which $\mathrm{KBs}$ function is to provide a link between researchers and practitioners, decision-makers and policy-makers [14], whereas another is to link knowledge users to the scientific literature while supporting the development of capacity for EIDM [12, 15]. KBs assess the need for organisational change, develop strategies to facilitate change and work to create a culture that values the use of the best available evidence in decision-making $[9,16]$. They are instrumental in facilitating communication and knowledge sharing among key stakeholders [17], facilitating learning [18-21], building capacity to locate, appraise and translate evidence into the local context $[18,22,23]$, and improving interpretation of research findings and development of implications for action [24]. While building individual knowledge, skills and capacity for EIDM, KBs also support the development of organisational context and infrastructure that supports emerging EIDM behaviours among staff $[12,25]$. It is believed that KBs must possess exceptional interpersonal, communication and motivational skills, as well as expertise from both the knowledge user's perspective and that of the research community to be effective [26-28].

In partnership with three Canadian public health departments, we used a case study design to conduct this study. The purpose of this study was to enhance individual capacity for EIDM and facilitate organisational contexts conducive towards it. Through the use of a case study methodology, we evaluated the impact of a KT intervention on EIDM knowledge, skills and behaviour, and identified contextual factors that facilitated or impeded impact. In this paper, we provide a thorough description of the KT intervention implemented by KBs in the three public health departments. Details related to the activities that were conducted and how they were implemented are provided such that others may replicate the intervention in other settings. The level of detail goes beyond what is generally included in publications reporting intervention outcomes. However, greater knowledge of what the intervention was comprised of and issues specifically related to implementation are needed in the $\mathrm{KT}$ literature. The primary focus of this paper is therefore to discuss important aspects related to intervention implementation and reflect on key factors that either facilitated or hindered its implementation.

\section{Methods}

\section{Participants}

This case study was funded by the Canadian Institutes of Health Research as part of the Partnerships for Health System Improvement competition (FRN101867). Participants included front-line public health practitioners, managers, directors, and Medical and Associate Medical Officers of Health from three public health departments (cases). Ethics approval was received from the McMaster University Research Ethics Board.

\section{Setting}

The three cases were situated in southwestern Ontario and differed with respect to the number of full-time staff, size of population served and organisational structure. They all served both urban and rural areas, as well as ethnically diverse populations, and were organised in discrete divisions or directorates such as communicable disease, chronic disease prevention and environmental health. Additional details related to the three cases are presented in Dobbins et al. 2018 [29]. 


\section{KT intervention}

The KT intervention targeted six of the seven steps in the evidence-informed public health process described by the NCCMT [30]. This included identifying a practice-based question, developing and conducting a search for relevant evidence, appraising the methodological quality of the retrieved evidence, synthesising the evidence, assessing the applicability of the evidence to the local context, and determining if changes to local public health policy and practice were necessary as a result of reviewing the evidence. A KB implemented the $\mathrm{KT}$ intervention in each case through large and small group interactive workshops, one-to-one mentoring through the process of conducting a rapid evidence review via face-to-face, electronic and telephone communication, attending meetings and presentations with senior management, and assisting in the development of policies and procedures that supported EIDM. The research team worked with each case to determine what form of interaction was preferred between the $K B$ and staff (i.e. amount of time and in what manner - one-toone versus small or large-group training).

The intervention was tailored for each case taking into account the philosophical stance, existing processes and supports, EIDM knowledge and skill of staff, and organisational goals. The intervention was implemented over
22 months. Each case was assigned a KB. While the KBs were employed by McMaster University, they spent a substantial amount of time located at the case sites. Given the time required to deliver the $\mathrm{KT}$ intervention, it was necessary to have two $\mathrm{KBs}$, where one was assigned two of the cases and the other $\mathrm{KB}$ was assigned one case. Both KBs held a Masters degree and had several years' experience working in $\mathrm{KT}$ and/or public health. In each case, the intervention began with an organisational assessment, followed by intervention implementation and ongoing monitoring and refinement of the intervention throughout the study. Additional details about the organisational assessment and results of this assessment have been published previously [29]. A more detailed description of the KT intervention implemented in each case is provided in Table 1.

\section{Ongoing monitoring and refinement of the $K T$ intervention}

The KBs, research team and senior management met regularly throughout the study to discuss intervention implementation and progress. During these discussions, ideas about how to continually modify the intervention arose and, in most instances, they were implemented. Case A identified new tools it wanted to use to support specific steps in the rapid evidence review process and revised existing tools. At the organisation level, Case A

Table 1 Details of the knowledge translation intervention, tailored for each health department

\begin{tabular}{|c|c|c|c|}
\hline & Case A & Case B & Case $\mathrm{C}$ \\
\hline Context & $\begin{array}{l}\text { Large, diverse population served; Medical } \\
\text { Officer of Health had vision for EIDM; EIDM } \\
\text { a strategic priority; committed resources }\end{array}$ & $\begin{array}{l}\text { Large, urban centre served; Medical Officer } \\
\text { of Health strongly committed to EIDM; } \\
\text { manager 'champion' for EIDM; EIDM a } \\
\text { strategic priority }\end{array}$ & $\begin{array}{l}\text { Mid-size, urban/rural mix served; Medical } \\
\text { Officer of Health committed to EIDM; } \\
\text { commitment from Executive team }\end{array}$ \\
\hline Intervention & September 2010 - June 2012 & April 2011 - February 2013 & April 2011 - December 2012 \\
\hline $\begin{array}{l}\text { KB on-site vs. } \\
\text { virtual }\end{array}$ & $\begin{array}{l}\text { 100\% on-site ( } 2 \text { days/week onsite for } 22 \\
\text { months) }\end{array}$ & $\begin{array}{l}25 \% \text { on-site; } 75 \% \text { virtual ( } 2 \text { days/month } \\
\text { onsite for first } 6 \text { months, then } 1 / 2 \text { day/week } \\
\text { for } 16 \text { months) }\end{array}$ & $\begin{array}{l}25 \% \text { on-site; } 75 \% \text { virtual ( } 2 \text { days/month } \\
\text { onsite for first } 6 \text { months, then } 1 \text { day every } \\
\text { other week for } 16 \text { months) }\end{array}$ \\
\hline $\begin{array}{l}\text { Organisational- } \\
\text { level strategies }\end{array}$ & $\begin{array}{l}\text { Workshop training for all staff; and KB } \\
\text { participated in intra-department } \\
\text { presentations }\end{array}$ & $\begin{array}{l}\text { Introductory workshops provided to } \\
\text { consultants; EIDM training for all staff in } \\
\text { one directorate }\end{array}$ & $\begin{array}{l}\text { Department-wide EIDM training through } \\
\text { large group workshops; KB advised } \\
\text { Research and Knowledge Exchange } \\
\text { Committee on creation of an EIDM } \\
\text { guidebook; development of an EIDM } \\
\text { organisational policy }\end{array}$ \\
\hline
\end{tabular}

\footnotetext{
Group-level KB mentored staff teams through rapid evidence reviews and provided EIDM training strategies

Individual-level KB mentored individual staff and was available for ad-hoc EIDM-related questions strategies

Senior Regular meetings and presentations to Management Senior Management Team interaction

Total activities 18 rapid evidence reviews, large-scale training sessions, KB facilitated/contributed to Critical Appraisal Club and presentations of research to staff colleagues and Senior Management

KB advocated for staff time to be allocated to EIDM and advised Senior Management Team

5 rapid evidence reviews; additional divisional training delivered; abstracts submitted to present research; presentations to Senior Management
}

$\mathrm{KB}$ advised Executive team on EIDM policy and procedures

5 rapid evidence reviews; EIDM policy and procedures developed and approved; presentations to Executive team; all-staff training delivered 
identified that additional strategies were needed to clarify staff roles and responsibilities with respect to EIDM. The KB also worked on developing workshop content and delivering EIDM training for all staff.

In Case B, an 'EIDM roadmap' was developed to help support and sustain EIDM. Identified responsibilities included developing and implementing brokering strategies, recommending communication within or across directorates to help avoid duplication of rapid evidence reviews or supporting work, clarifying roles in EIDM broadly and for KB work specifically, helping the health department negotiate a selection of priority questions, and generating practical recommendations for internal consultants.

Case C implemented an EIDM policy that was ultimately endorsed and adopted by the health department's Executive Committee. With approval from senior management, the purpose of an existing committee was revised to include responsibility for developing and implementing strategies to sustain EIDM in the organisation. This health department also implemented voluntary basic EIDM training for all employees comprised of a full day EIDM introductory workshop and a subsequent half day workshop on critical appraisal of systematic reviews.

\section{Evaluation method and measures}

An estimate of intervention dose (i.e. overall time and type) and intensity according to level (i.e. organisation, group, individual) was tracked by the KBs in a reflective journal. KBs maintained a daily reflective journal of activities undertaken, as well as observations and reflections of the impact of these activities on EIDM. KBs also documented key factors that either facilitated or hindered implementation. Data from the KBs' reflective journals were entered in NVIVO 8. The data were coded for emerging trends and themes by several members of the research team (RT, LG, KD, MD). Once agreement on major themes and codes were reached, and a coding scheme developed, the data were coded by one member of the research team (RT). The research team met to discuss interpretation of the themes.

\section{Results}

The KBs spent 2 days per week delivering the KT intervention in each case, although there were substantial differences in how the time was allocated. In Case A, the KB was physically onsite 2 days per week for the full 22 months. The KB spent the majority of time mentoring teams through rapid evidence reviews, and assessing the applicability of the research evidence to the local setting. The KB also participated in a monthly critical appraisal club with staff, provided workshops on EIDM, responded to questions posed by staff related to EIDM, and attended and presented at meetings with senior management and other health department staff. Presentations included an introduction to EIDM, interpreting statistics, and searching for and critically appraising public health practice guidelines. The total number of staff who were mentored, either individually or in small groups by the $\mathrm{KB}$, was 48 , whereas 33 staff participated in large group training such as presentations or workshops.

In Case B, the KB was initially physically onsite 2 days during 1 week of the month, but after approximately 6 months, at the request of the organisation, this was changed to half a day each week. The organisation indicated having the KB onsite more frequently would help maintain momentum for those engaged with the $K B$. The KB spent time onsite engaged in two specific activities, namely (1) building capacity for EIDM among staff who had responsibility for incorporating research evidence into practice decisions; and (2) conducting workshops with other staff about what EIDM is and why it is important. The KB also attended and presented at meetings with the senior management team and other health department staff. Twelve staff were mentored either individually or in small groups, by the KB; 76 participated in large group training such as presentations or workshops.

In Case $\mathrm{C}$, the $\mathrm{KB}$ also started out being physically onsite 2 days during 1 week of the month, but 6 months into the intervention, the case requested this be changed to 1 day every other week. The KB spent more time conducting large group training workshops, although small groups were also mentored through rapid evidence reviews. The $\mathrm{KB}$ also worked with the Research and Knowledge Exchange committee to develop policies, procedures and a guidebook for EIDM, and presented at senior management meetings and department-wide symposia. The total number of staff that engaged either individually or in small groups with the KB was 17 , whereas 49 participated in large group training such as presentations or workshops.

Overall, a statistically significant improvement in knowledge and skill was observed from baseline to follow-up in each case. Further details and discussion of these results have been reported elsewhere [29]. The remainder of this paper focuses on the results of observations from implementing the intervention through assessment of the KBs' reflective journals.

\section{Implementation facilitators and challenges}

The following are the key factors - at the organisational, team and individual level - perceived by the KBs that either facilitated or hindered implementation of the KT intervention and, ultimately, EIDM. 


\section{Facilitators}

Particularly important factors in supporting EIDM at the organisational level were strong leadership and support for changing culture and practice to systematically incorporate research evidence into the decision-making process. Champions to communicate the value of EIDM supported 'getting the word out' and increased knowledge and understanding among staff about what EIDM is and is not. Existing infrastructure, such as a library and on-staff librarians, was identified as a key factor that enabled EIDM, as well as having particular roles identified in the organisation with responsibility for finding and using research evidence. Each case recognised the existence of committed financial and human resources, willingness to participate in the research study and support for staff to spend time engaged in the EIDM process as fundamental to the EIDM process. Existing EIDM processes and tools, such as those from the NCCMT and Health Evidence, helped the cases adopt or develop their own EIDM policies, procedures and tools. An important component of the KT interventions, observed by the $\mathrm{KBs}$ as contributing to successful EIDM, was regular contact between the KBs, and KBs and staff, research team, case key contact, and senior management.

At the team level, the KBs identified meaningful interest in EIDM among teams and enthusiasm to improve knowledge and skills in finding and using research evidence in decision-making as important factors. This was observed especially among teams that were working on resolving important priority policy or programme decisions in their health department. The KBs noted that small teams working on rapid evidence reviews on topics of relevance and importance to the health department were seen among their peers as 'taking small steps', which enabled the health department to pilot EIDM processes and 'test' whether EIDM was feasible and valuable for making important practice decisions. In some cases, teams that were already skilled in finding and using research but who wanted to formalise their processes, were instrumental in demonstrating the feasibility and usefulness of EIDM. Sharing early experiences reassured and provided support for teams who later engaged in EIDM.

At the individual level, the KBs noted that curiosity and enthusiasm among staff supported culture change and the creation of EIDM processes and practices. The KBs indicated that identifying staff with existing EIDMrelated training and experience and getting them involved in the intervention was helpful, particularly in the early stages of implementing the KT intervention.

\section{Challenges}

The KBs observed that challenges to implementing the intervention mirrored facilitators to a large extent. For example, without leadership, champions, infrastructure and committed resources, the KBs noted that the cases struggled to achieve culture and practice changes that formally and systematically incorporated research evidence into decision-making. At the organisational level, KBs commented that limited engagement in and modelling of EIDM behaviours by senior management tended to result in similar behaviours by staff throughout the organisation. The KBs reflected that organisational change required active involvement of leadership that was consistently observable throughout the organisation. They also noted that verbal communication of the organisation's commitment to EIDM was insufficient in facilitating organisational change.

At the team level, the KBs observed how managers could act as barriers to staff development with EIDM. In instances where managers lacked understanding of the EIDM process or the time required to develop skills for EIDM and conduct activities consistent with EIDM, KBs observed staff did not have adequate time allocated to these tasks. As a result, staff struggled to make progress and/or experienced pressure to complete their work more quickly. Further, when teams reported their public health work as 'mandated' or externally prescribed, it was challenging to engage staff in EIDM as they did not perceive opportunities existed to change practice. This significantly reduced interest and motivation to learn EIDM-related skills across some teams and divisions/ directorates.

At the staff level, individuals varied greatly with respect to the amount of EIDM-related training they required to integrate EIDM in day-to-day work. This presented challenges with respect to developing multiple unique training programmes at the individual and team levels. Other challenges included occasional resistance to participation in EIDM and to changing practice status quo. A lack of confidence among staff regarding EIDMrelated knowledge and skill was consistently identified. Most challenging was the reality of competing priorities and lack of time. This was sometimes characterised as staff not seeing EIDM as part of 'core work', but rather an 'add-on' to their already full workloads.

\section{Discussion}

Each case involved in this study featured a unique combination of individual, team and organisational characteristics and circumstances relating to their communities, political contexts and available resources. Furthermore, they were involved, to varying extents, in supporting EIDM prior to this study and therefore may have been more amenable to EIDM adoption in comparison to other public health departments in Ontario and Canada. Each case was involved in designing its own KT intervention, which has been shown in other studies to be associated with a higher degree of uptake of research evidence [31]. 
As part of the KT intervention, KBs actively helped public health professionals identify, appraise and interpret best available research evidence as well as assess its applicability to the local context and determine if practice changes were needed based on review of the evidence. Previous evidence has shown that active engagement of decisionmakers in applying evidence to practice improves uptake of research evidence [6].

The findings of this study are supported by others who have reported that $\mathrm{KT}$ interventions need to be tailored to the specific context of organisations and the health professionals working within them $[6,28]$. Furthermore, the complexity of the KT intervention implemented in this study is also supported by others who have suggested that EIDM is much more complex than previously thought and that it is likely that single, one-off interventions such as workshops or journal clubs are insufficient to realise EIDM in health organisations, including public health [32, 33]. Current thinking supports an organisation-wide approach to EIDM [33].

A variety of change agents have been used to implement KT interventions, including facilitators [34, 35], opinion leaders $[9,36]$, academic detailers and KBs. KBs have been shown previously to promote and enhance EIDM [9] and to be adaptable to different contexts [22]. In this study of public health departments, the KT intervention implemented by KBs was associated with statistically significant improvements in EIDM knowledge, skills and behaviours [29]. Others have reported that KT interventions implemented by KBs were effective in facilitating and improving communication and knowledge sharing between key stakeholders [17], in building capacity to locate, appraise and translate evidence to the local context, and in increasing skill to interpret and apply research evidence to the practice setting $[19,21]$.

In this case study, particular activities were highly important at the outset of the intervention in setting the stage for intervention implementation; specifically, plans to draft a policy and procedures guidebook, with subsequent rapid evidence review experience that informed the tools for a guidebook, meetings with the Medical Officers of Health, and development of a document outlining a process for change at both the team and organisation levels. These activities acted as catalysts in each of the health departments. KBs have been shown previously to be catalysts for systems change [17], as well as establishing and nurturing connections within organisations [28]. Questions remain, however, with respect to optimal timing for intervention initiation and how to sustain EIDM in the organisation(s).

The KBs in this study supported participants to gain capacity in locating, appraising, translating and assessing applicability and applying evidence to practice, as well as assisting each case to improve its EIDM processes. The
KBs identified librarians in two of the cases as champions for EIDM, while the third case identified library services as a barrier to EIDM [37]. Other factors identified as important to EIDM by the KBs included available resources, participation of the local community, cultural appropriateness, and social and political issues [38]. The KBs acted as mentors, gradually transferring responsibility to decision-makers as skills improved, eventually enabling staff to work with minimal supervision or support. Others have reported similar findings with respect to the $\mathrm{KB}$ role in implementing $\mathrm{KT}$ interventions as evolving over time as knowledge and skill among the workforce improves [22, 24].

The findings of this study demonstrate an increase in EIDM knowledge and skills among staff and improvement in EIDM behaviours, particularly among staff who interacted closely with the KBs. Findings also highlight the importance of infrastructure and policies aimed at maintaining and sustaining a culture of EIDM, which has also been reported in the literature [25]. Tangible structures and resources, which were identified in this study as playing a crucial role in EIDM, have also been reported by others to be an important precursor to organisational change [39]. The findings of this study suggest that tailored $\mathrm{KT}$ interventions implemented in public health departments are promising for encouraging and supporting EIDM. The findings of this study also suggest that the intervention can lead to impact when implemented by KBs in public health departments. However, questions remain as to how setting and context affect the impact of change agents on EIDM, despite the publication of multiple studies on this topic over the past 10 years. Additional research is needed to more fully understand the impact of these various changeagent roles, as well as concepts related to organisational readiness for change, supporting culture change, attaining leadership commitment to EIDM, and how setting and context affect change-agent impact.

\section{Conclusions}

The results of this study illustrate the importance of working collaboratively with organisations to tailor $\mathrm{KT}$ interventions to best meet unique needs, preferences, organisational structures and contexts. Organisational factors, such as leadership, commitment to culture change, available resources and infrastructure, are critical in supporting the change process. Public health departments considering embarking on a process to embed EIDM into routine practice should first identify organisational strengths for EIDM, and work to optimise organisational supports where they exist and develop them where they do not. Once these are in place, organisations are more likely to be ready for change and to engage actively in developing and implementing KT strategies. While KBs 
implemented the KT intervention in this study, more research is needed to understand the impact of all changeagent roles, including KBs, as well as how these roles can be maintained in the long-term if proven effective.

\section{Abbreviations}

EIDM: evidence-informed decision-making; KB: knowledge broker KT: knowledge translation; NCCMT: National Collaborating Centre for Methods and Tools

\section{Acknowledgements}

A sincere thank you to the KBs who painstakingly documented the KT intervention and reflected on the impact of their activities along the way. We are also indebted to the public health departments that participated with us on this journey toward EIDM.

\section{Authors' contributions}

MD conceptualised the study, was involved in all aspects of study implementation, contributed to drafts of the paper, and finalised the manuscript for submission for publication. LG wrote the first draft of the manuscript and contributed to subsequent drafts. JY assisted with conceptualisation of the first draft of the paper and reviewed drafts of the manuscript. RT assisted in the writing of the first draft and reviewed subsequent drafts. KD-W was one of the KBs who delivered the intervention. She contributed to analysis of the KB's reflective journals and reviewed drafts of the paper. RY-N provided statistical consultation to the project and reviewed drafts of the manuscript. All authors read and approved the final manuscript.

\section{Authors' information}

Dobbins has been working with the public health community for over two decades to identify, develop, implement and evaluate knowledge translation interventions to support evidence-informed decision-making.

\section{Funding}

Funding was obtained from the Canadian Institutes of Health Research, Partnerships for Health System Improvement initiative (FRN 101867). The study funder, the Canadian Institutes of Health Research, had no role in the study's design, data collection, analysis or interpretation, or writing of this manuscript.

\section{Availability of data and materials}

Data for this study are housed on a password-protected server at McMaster University, and available upon request from the primary author.

\section{Ethics approval and consent to participate}

Ethics approval for this study was obtained from the McMaster University Faculty of Health Sciences Research Ethics Board.

\section{Consent for publication}

Not applicable.

\section{Competing interests}

The authors declare that they have no competing interests.

\section{Author details}

${ }^{1}$ Faculty of Health Sciences, School of Nursing, McMaster University, 175 Longwood Road South, Suite 210A, Hamilton, Ontario, Canada. ${ }^{2}$ Region of Peel - Public Health, 7120 Hurontario Street, Mississauga, Ontario, Canada. ${ }^{3}$ M. Louise Fitzpatrick College of Nursing, Villanova University, Driscoll Hall, Room 330, 800 Lancaster Avenue, Villanova, PA, United States of America. ${ }^{4}$ Department of Community Health and Epidemiology, Dalhousie University, Halifax, Nova Scotia, Canada. ${ }^{5}$ Centre for Person-Centered Practice Research, Queen Margaret University Edinburgh and Affiliate Member, Queen Margaret University, Edinburgh, United Kingdom. ${ }^{6}$ Department of Public Health Sciences, University of Rochester, School of Medicine, 265 Crittenden Blvd., CU 420644, Rochester, New York 14642, United States of America.
Received: 20 August 2018 Accepted: 15 May 2019

Published online: 20 June 2019

\section{References}

1. Ontario Public Health Standards. Minister of Health and Long-Term Care. 2008. http://www.ontla.on.ca/library/repository/mon/23007/285251.pdf. Accessed 1 Apr 2018.

2. Brownson RC, Fielding J, Maylahn C. Evidence-based public health: a fundamental concept for public health practice. Annu Rev Public Health. 2009;30(1):175-201.

3. Straus SE, Tetroe JM, Graham ID. Knowledge translation is the use of knowledge in health care decision making. J Clin Epidemiol. 2011; 64(1):6-10.

4. Ciliska D. Introduction to evidence-informed decision making. In: Canadian Institutes of Health Research; 2012. http://www.cihr-irsc.gc.ca/ e/45245.html. Accessed 15 Apr 2018.

5. Hausman AJ. Implications of evidence-based practice for community health. Am J Community Psychol. 2002;30(3):453-67.

6. Lavis J, Davies H, Oxman A, Denis JL, Golden-Biddle K, Ferlie E. Towards systematic reviews that inform health care management and policy-making J Health Serv Res Policy. 2005;10(3 Suppl. 1):35-48

7. Dobbins M, Hanna SE, Ciliska D, Thomas H, Manske S, Cameron R, Mercer S, O'Mara L, DeCorby K, Robeson P. A randomized controlled trial evaluating the impact of knowledge translation and exchange strategies. Implementation Sci. 2009;4(1):61.

8. Toomey T, Tramel S, Erickson D, Lenk K. Use of research based information among leaders of public health agencies. Am J Health Educ. 2009;40(2):66.

9. Lomas J. The in-between world of knowledge brokering. BMJ. 2007; 334:129-32.

10. Bowen S, Martens P. Demystifying knowledge translation: learning from the community. J Health Serv Res Policy. 2005;10(4):203-11.

11. Dobbins M, Robeson P, Ciliska D, Hanna S, Cameron R, O'Mara L, DeCorby K, Mercer S. A description of a knowledge broker role implemented as part of a randomized controlled trial evaluating three knowledge translation strategies. Implement Sci. 2009:4:23.

12. Conklin J, Lusk E, Harris M, Stolee P. Knowledge brokers in a knowledge network: the case of Seniors Health Research Transfer Network knowledge brokers. Implement Sci. 2013;8:7.

13. Ward $\mathrm{VL}$, House AO, Hamer S. Knowledge brokering: exploring the process of transferring knowledge into action. BMC Health Serv Res. 2009;9:12.

14. Bornbaum CC, Kornas K, Peirson L, Rosella LC. Exploring the function and effectiveness of knowledge brokers as facilitators of knowledge translation in health-related settings: a systematic review and thematic analysis. Implement Sci. 2015;10(1):162.

15. Cameron D, Russell DJ, Rivard L, Darrah J, Palisano R. Knowledge brokering in children's rehabilitation organizations: perspectives from administrators. J Contin Educ Heal Prof. 2011:31(1):28-33.

16. Gagnon ML. Moving knowledge to action through dissemination and exchange. J Clin Epidemiol. 2011;64(1):25-31.

17. Lyons $R$, Warner $G$, Langille $L$, Phillips SJ. Piloting knowledge brokers to promote integrated stroke care in Atlantic Canada. In: Moving Population and Public Health Knowledge into Action: A Casebook of Knowledge Translation Stories. Ottawa: Canadian Institutes of Health Research (CIHR) Institute for Population and Public Health; 2006. https://www.cfhi-fcass.ca/ SearchResultsNews/08-01-01/7d6652f7-c825-4ea5-9797-8ff958c5f962.aspx. Accessed 12 June 2019

18. Hartwich F, von Oppen M. Knowledge brokers in agricultural research and extension. In: Graef F, Lawrence P, von Oppen M, editors. Adapted Farming in West Africa: Issues, Potentials, and Perspectives. Stuttgart: Verlag Ulrich E. Grauer; 2000. p. 445-53.

19. Loew R, Bleimann U, Walsh P. Knowledge broker network based on communication between humans. Campus-Wide Inf Syst. 2004:21(5):185-90.

20. Dobbins $M$, DeCorby $K$, Twiddy $T$. A knowledge transfer strategy for public health decision makers. Worldviews Evid-Based Nurs. 2004;1(2):120-8.

21. Hinloopen J. The market for knowledge brokers. Small Bus Econ. 2004;22: 407-15.

22. Pyper C. Knowledge brokers as change agents. In: Lissauer R, Kendall L, editors. New Practitioners in the Future Health Service: Exploring Roles for Practitioners in Primary and Intermediate Care. London: Institute for Public Policy Research; 2002. p. 60-70. 
23. Lavis JN, Robertson D, Woodside J, McLeod C, Abelson J. How can research organizations more effectively transfer research knowledge to decision makers? Milbank Q. 2003;81(2):221-48.

24. Thompson GN, Estabrooks CA, Degner LF. Clarifying the concepts in knowledge transfer: a literature review. J Adv Nurs. 2006;53(6):691-701.

25. Van Kammen J, De Savigny D, Sewankambo N. Using knowledge brokering to promote evidence-based policy-making: the need for support structures. Bull World Health Organ. 2006;84(8):608-12.

26. Urquhart R, Porter GA, Grunfeld E. Reflections on knowledge brokering within a multidisciplinary research team. J Contin Educ Heal Prof. 2011;31(4): 283-90.

27. Greenhalgh T, Robert G, MacFarlane F, Bate P, Kyriakidou O. Diffusion of innovations in service organizations: systematic review and recommendations. Milbank Q. 2004;82(4):581-629.

28. Choi BCK, Pang T, Lin V, Puska P, Sherman G, Goddard M, Ackland M Sainsbury P, Stachenko S, Morrison H, Clottey C. Can scientists and policy makers work together? J Epidemiol Community Health. 2005;59(8):632-7.

29. Dobbins M, Traynor RL, Workentine S, Yousefi-Nooraie R, Yost J. Impact of an organization-wide knowledge translation strategy to support evidence-informed public health decision making. BMC Public Health. 2018;18(1):1412

30. National Collaborating Centre for Methods and Tools. An Introduction to Evidence-Based Public Health and a Compendium of Critical Appraisal Tools for Public Health Practice (Revised). https://www.nccmt.ca/uploads/media/ media/0001/01/b331668f85bc6357f262944f0aca38c14c89c5a4.pdf. Accessed 2 Jan 2018.

31. Canadian Health Services Research Foundation. Issues in Linkage and Exchange between Researchers and Decision Makers. 1999. https:// www.cfhi-fcass.ca/migrated/pdf/event_reports/linkage_e.pdf. Accessed 10 Mar 2018.

32. Taylor RS, Reeves BC, Ewings PE, Taylor RJ. Critical appraisal skills training for health care professionals: a randomized controlled trial. BMC Med Educ. 2004:4:30.

33. Jewell CJ, Bero LA. "Developing good taste in evidence": facilitators of and hindrances to evidence-informed health policymaking in state government. Millbank Q. 2008;86(2):177-208.

34. Ellis I, Howard P, Larson A, Robertson J. From workshop to work practice: an exploration of context and facilitation in the development of evidencebased practice. Worldviews Evid-Based Nurs. 2005;2(2):84-93.

35. Burtonwood AM, Hocking PJ, Elwyn G. Joining them up: the challenges of organisational change in the professional politic of general practice. J Interprof Care. 2001;15(4):383-93.

36. Sisk JE, Lennarson Greer A, Wojtowycz M, Pincus LB, Aubry RH. Implementing evidence-based practice: evaluation of an opinion leader strategy to improve breast feeding rates. Am J Obstet Gynecol. 2004; 190(2):413-21.

37. Yousefi Nooraie R, Marin A, Hanneman R, Lohfeld L, Dobbins M. Implementation of evidence-informed practice through central network actors; a case study of three public health units in Canada. BMC Health Serv Res. 2017;17:208

38. Banta HD. Considerations in defining evidence for public health. Int J Technol Assess Health Care. 2003;19(3):559-71.

39. McCormack B, Rycroft-Malone J, DeCorby K, Hutchinson AM, Bucknall T, Kent B, Schultz A, Snelgrove-Clarke E, Stetler C, Titler M, Wallin L, Wilson V. A realist review of interventions and strategies to promote evidence-informed healthcare: a focus on change agency. Implement Sci. 2013;8:107.

\section{Publisher's Note}

Springer Nature remains neutral with regard to jurisdictional claims in published maps and institutional affiliations.

\section{Ready to submit your research? Choose BMC and benefit from}

- fast, convenient online submission

- thorough peer review by experienced researchers in your field

- rapid publication on acceptance

- support for research data, including large and complex data types

- gold Open Access which fosters wider collaboration and increased citations

- maximum visibility for your research: over $100 \mathrm{M}$ website views per year

At BMC, research is always in progress.

Learn more biomedcentral.com/submissions 